\title{
Percepção dos profissionais sobre a coordenação entre níveis de atenção à saúde em dois municípios pernambucanos de grande porte
}

\author{
Renata Patrícia Freitas Soares de Jesus(a) \\ Antônio Carlos Gomes do Espírito Santo(b) \\ Marina Ferreira de Medeiros Mendes ${ }^{(c)}$ \\ Isabella Chagas Samico(d)
}

Jesus RPFS, Espírito Santo ACG, Mendes MFM, Samico IC. Health care professionals' perceptions of coordination between levels of care in two large municipalities in the State of Pernambuco, Brazil. Interface (Botucatu). 2018; 22(65):423-34.

This study aimed to gain insight into healthcare professionals' perceptions of coordination between levels of care in two municipalities in the State of Pernambuco, Brazil. A qualitative study was conducted consisting of in-depth interviews with 51 professionals working in primary care (PC) and secondary care (SC) regarding clinical management and the factors affecting its implementation. The results show that, despite a lack of understanding of the term coordination across levels of care, the importance of and efforts focused on this area are recognized. Lack of communication between PC and SC is viewed as the cause and consequence of problems that hamper the successful implementation of clinical governance across the dimensions of follow-up care, accessibility, and consistency in care delivery. Initiatives to improve communication between PC and SC professionals in order of promoting mutual knowledge, strenghten professional qualification and joint management of cases were not observed.

Keywords: Health care. Levels of health care. Clinical management. Qualitative research.
Objetivou-se conhecer a percepção de profissionais de saúde sobre a coordenação da atenção entre níveis assistenciais em dois municípios do estado de Pernambuco, Brasil. Pesquisa qualitativa foi realizada por meio de entrevistas em profundidade, com 51 profissionais de atenção básica $(A B)$ e especializada (AE) quanto à coordenação da gestão clínica e fatores que a influenciam. Os resultados mostraram que, embora se observe compreensão insuficiente do termo Coordenação da atenção entre níveis, há reconhecimento da importância e do esforço em investir nesse processo. A insuficiência de comunicação entre $A B$ e $A E$ é vista como causa e consequência de problemas que dificultam a coordenação da gestão clínica nas dimensões do seguimento, acessibilidade e coerência da atenção. Não se observaram iniciativas de aproximação entre profissionais da $A B$ e $A E$, no sentido de promover conhecimento mútuo, fortalecer a qualificação profissional e o manejo conjunto dos casos.

Palavras-chave: Atenção à saúde. Níveis de atenção à saúde. Gestão clínica. Pesquisa qualitativa.

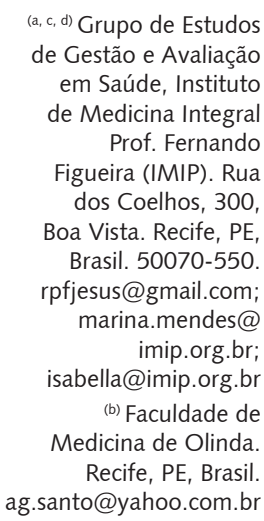




\section{Introdução}

Entre os países latino-americanos, a exemplo do que acontece em todo o mundo, a coordenação do cuidado é tida como componente essencial de uma atenção primária (APS) abrangente ${ }^{1-3}$. É um conceito complexo e multidimensional, está entrelaçado com a continuidade e a qualidade da atenção e recebe diferentes definições ${ }^{4-6}$. Para Starfield ${ }^{7}$, a coordenação é sinônimo de integração dos serviços, mas pode ser compreendida como a articulação dos diversos serviços e ações relacionados à atenção, voltada ao alcance de uma resposta integral6,8. Esta, para alguns autores ${ }^{9,10}$, só é possível se 0 cuidado for organizado em redes, definidas como organizações poliárquicas de conjuntos de serviços, vinculados entre si por objetivos comuns e por uma ação cooperativa e interdependente que permitem ofertar uma atenção contínua e integral coordenada pela APS. A atenção deve ser prestada no tempo e lugar certo, a um custo corretamente estipulado, com qualidade aceitável, de forma humanizada e com responsabilidade sanitária e econômica ${ }^{11}$.

Estudos sobre coordenação do cuidado podem envolver a integração entre serviços em determinado nível de atenção ou entre diferentes níveis, sendo esta última a abordagem assumida neste estudo, para o qual a coordenação foi tomada como a conexão harmoniosa dos diferentes serviços necessários para cuidar de um paciente na sequência dos cuidados ${ }^{12-14}$.

A fragilidade na coordenação entre níveis mostra-se mais evidente na atenção aos pacientes com doenças crônicas, que requerem múltiplos profissionais e diversos serviços. Dentre tais doenças, as não transmissíveis (DCNT) representam um grave problema de saúde pública, sendo responsáveis por $68 \%$ das mortes no mundo. O Brasil acompanha esta tendência, onde as DCNT respondem por $74 \%$ das mortes, destacando-se: as cardiovasculares (31\%), cânceres (17\%), respiratórias crônicas (6\%) e diabetes $(6 \%)^{15}$.

Mendes ${ }^{11}$ chama a atenção para o descompasso entre as demandas epidemiológicas do século XXI e a capacidade de resposta dos sistemas de saúde, estruturados a partir de um contexto sanitário prevalente nas primeiras décadas do pós-guerra. Para fazer frente a tal realidade, reformas nacionais vêm sendo promulgadas, com diferenças entre elas, seja no que diz respeito: ao momento de implantação, às estruturas de rede, aos mecanismos de coordenação ou aos resultados obtidos na qualidade dos serviços, dos indicadores de saúde, da satisfação dos usuários, e no controle dos custos $^{16-18}$.

No Brasil, os entraves verificados para a efetivação das medidas para integração das redes têm sido atribuídos a diversos fatores, dentre eles: a insuficiência de investimentos necessários à conquista do acesso universal ${ }^{16} \mathrm{e}$ à forma como se dá o processo de descentralização da saúde, marcada por uma autonomia atomizada dos municípios ${ }^{17}$. A Estratégia Saúde da Família, que exerce um papel central na ordenação do acesso aos diferentes níveis do sistema, alcançou expressivo aumento de cobertura, mas não sanou a fragilidade dos mecanismos de referência entre os níveis de atenção ${ }^{19}$.

Atualmente, após mais de 25 anos de implantação do SUS, o acesso com integralidade continua sendo um problema a superar e a coordenação entre níveis desempenha importante papel nesse contexto. É possível que o estágio de institucionalização dessa coordenação tenha diferentes graus em cada região do Brasil, e que fatores locais e regionais interfiram, num país tão extenso e desigual. Nesse sentido, a ótica dos atores que atuam nos diversos componentes da estrutura das redes representa fonte de informação relevante.

Nesta pesquisa foram estabelecidas, como objeto de estudo, as redes de atenção à saúde de dois municípios de grande porte do estado de Pernambuco; e como objetivo, conhecer a percepção de ocupantes de cargos administrativos e de profissionais de saúde que desempenham funções assistenciais, sobre a coordenação entre níveis de atenção.

\section{Percurso metodológico}

A investigação, de natureza qualitativa, utilizou dados da pesquisa: "Impacto das estratégias de integração da atenção no desempenho das redes de serviços de saúde em diferentes sistemas de 
saúde da América Latina - EquityLA II", um estudo multicêntrico financiado pela Comissão Europeia, como Ação de Cooperação Internacional Específica (SICA), e coordenado pelo Consórcio de Saúde da Catalunha. Esse estudo tem duração prevista de cinco anos (2014 - 2018) e objetiva avaliar a efetividade de diferentes estratégias de integração da atenção na melhoria da coordenação e da qualidade das redes de serviços de saúde em seis países da América Latina. No Brasil, as instituições parceiras são: o Instituto de Medicina Integral Prof. Fernando Figueira - IMIP e a Universidade de Pernambuco - UPE 20.

Os municípios brasileiros selecionados, aqui identificados como A e B, são de grande porte, ou seja, possuem acima de 100 mil habitantes, segundo o IBGE, 201121, e pertencem, respectivamente, às regiões agreste e metropolitana do estado de Pernambuco.

A amostra teórica foi definida em duas etapas:

Na etapa I, selecionaram-se unidades da atenção básica que atendiam a uma área geográfica específica da rede, situadas em área urbana com predominância de população de baixo nível socioeconômico. Para seleção das áreas, utilizaram-se como critérios: a taxa de densidade demográfica, o índice de desenvolvimento humano (IDH) e o interesse dos profissionais em participar da pesquisa. Posteriormente, foram selecionados os centros especializados de referência ao atendimento a pacientes com enfermidades crônicas.

Na etapa II, os critérios para selecionar os perfis dos informantes foram: a atuação i) na Atenção Básica ( $A B)$ e Especializada ( $A E)$ como médicos e enfermeiros há pelo menos seis meses; ii) na gestão em postos diretamente relacionados à coordenação entre níveis da atenção, tais como: diretores de distrito, gerentes de território, coordenadores de unidade de saúde; iii) em atividades de regulação e apoio institucional de território.

O tamanho da amostra final foi estabelecido com base no critério de saturação (Quadro 1).

Quadro 1. Composição da amostra de informantes para a análise da coordenação entre níveis de atenção em dois municípios de Pernambuco, 2014-2015.

\begin{tabular}{|c|l|c|c|}
\hline \multicolumn{2}{|c|}{} & Rede A & Rede B \\
\hline \multirow{3}{*}{ Entrevistas individuais } & Profissionais da Atenção Básica & 10 & 5 \\
\cline { 2 - 4 } & Profissionais da Atenção Especializada & 6 & 6 \\
\cline { 2 - 4 } & Diretores, Gerentes e Gestores & 6 & 6 \\
\cline { 2 - 4 } & Pessoal administrativo & 6 & 6 \\
\cline { 2 - 4 } & Total & $\mathbf{2 8}$ & $\mathbf{2 3}$ \\
\hline
\end{tabular}

Conduziram-se entrevistas individuais semiestruturadas utilizando-se guia tópico, com uma seção comum e uma seção específica para cada grupo informante.

O guia incluiu a opinião sobre a coordenação entre níveis nas redes de atenção e os fatores que a influenciam; e o papel dos níveis na atenção aos pacientes e sua relação com a coordenação entre eles.

As entrevistas foram conduzidas no local de trabalho dos informantes, em ambiente reservado, gravadas em áudio e transcritas na íntegra. A coleta transcorreu entre agosto de 2014 e janeiro de 2015.

Os dados foram processados e analisados de acordo com os procedimentos da análise temática de conteúdo, por meio do software ATLAS TI, e cada entrevista foi analisada por dois pesquisadores ${ }^{22}$. Os dados foram segmentados por município, grupo de informante e temas. A fim de se garantir a qualidade, a informação foi triangulada. Os resultados dos diferentes grupos de informantes foram contrastados entre si e com a literatura.

O projeto de pesquisa foi aprovado por Comitê de Ética em Pesquisas em Seres Humanos, protocolos No 2013/5048/I e 3967-14. 


\section{Resultados e discussão}

\section{Entendimento acerca da 'Coordenação da Atenção entre níveis'}

De modo geral, não foi demonstrada, pelos entrevistados, a compreensão do termo 'Coordenação da Atenção entre níveis' como sendo a articulação de todos os serviços relacionados com a atenção ao paciente, de modo harmônico, para a obtenção de um objetivo comum ${ }^{13,8}$. A expressão foi, inicialmente, entendida, pelos informantes, como sendo uma instância da cadeia hierárquica de gestão das organizações, necessitando, portanto, ser ressignificada pelos entrevistadores, por meio do uso de aproximações com termos como 'articulação ou integração da atenção básica com a atenção especializada'.

A partir de então, os profissionais informaram não perceber sua presença no cotidiano assistencial, ao passo que os ocupantes de cargos de gestão e gerenciamento defendiam a sua existência com base na ampliação da capacidade instalada e aumento da oferta de procedimentos, ainda que admitissem a necessidade de profundas melhorias para que pudesse tornar-se efetiva.

À medida que as indagações passaram a ser dirigidas aos tipos, dimensões e atributos da coordenação, identificou-se a existência de aspectos mais e menos críticos, a partir de julgamentos comparativos entre eles feitos pelos entrevistados ou com base na evolução desses aspectos ao longo da história recente de funcionamento das redes.

A expressão 'Coordenação da Atenção entre Níveis' é utilizada há mais de uma década na literatura internacional sobre APS e sobre a conformação de redes de atenção, estando presente em publicações científicas e em estudos emitidos por organismos sanitários ${ }^{7,23}$. No Brasil, embora o termo apareça em trabalhos como os de Almeida e Mendes ${ }^{8,24}$ e em alguns documentos oficiais ${ }^{25}$, a palavra integração tem sido o vocábulo mais frequentemente utilizado.

Conill et al. ${ }^{16}$ detectaram uma proliferação de conceitos que facilitam confusões e dificultam sínteses. Martinez et al. ${ }^{26}$, por sua vez, destacaram a variedade de conceitos sobre coordenação assistencial, o que dificulta a comparabilidade entre resultados obtidos em distintas regiões e países.

Da mesma maneira que observado no estudo de Almeida et al. ${ }^{8}$, identificou-se maior frequência de respostas acerca da não existência da coordenação na fala dos profissionais da assistência. Segundo Vargas et al. ${ }^{27}$, a formação profissional, que não valoriza a APS como ordenadora do cuidado, dificulta a coordenação em todo o contínuo do cuidado. Esse aspecto, assim como fragilidades na organização do processo de trabalho, contribuiriam não só para que os médicos da atenção básica deixem de exercer adequadamente suas responsabilidades, como, também, para que especialistas não estejam dispostos a colaborar devido à falta de conhecimento e ao pouco valor e confiança que depositam no nível responsável pelos cuidados primários.

\section{Sobre a coordenação da gestão clínica}

Ao lado da 'coordenação da informação' e da 'coordenação administrativa', a 'coordenação da gestão clínica' conforma os três tipos nos quais a coordenação da atenção é subdividida ${ }^{20,28}$. A coordenaçãa da gestão clínica se refere à oferta de atenção de maneira sequencial e complementar, segundo um plano de cuidados compartilhado pelos diferentes níveis de atenção que compreende as dimensões: 'seguimento adequado do paciente', 'acessibilidade' e 'coerência da atenção entre níveis assistenciais'.

O seguimento diz respeito ao acompanhamento do paciente, quando a natureza do episódio motiva o atendimento em diferentes níveis. Um de seus atributos refere-se à existência de um responsável clínico como coordenador desse processo de atenção.

Verificou-se que o discurso dos entrevistados no município A não revelou a identificação de tal responsável. "[...] acaba que o paciente não tem uma continuidade na assistência, ele é visto aqui de uma forma por um profissional e lá na atenção básica ele acaba sendo visto de outra forma.[...]. É como se ele tivesse sendo visto por pedaços né?!" (Apoio Institucional, AE, Município A). 
Também se observaram variadas compreensões acerca de quem deveria assumir a responsabilidade, não se estabelecendo consenso a respeito.

"[...] Eu acho que a responsabilidade técnica desse cuidado é da equipe de saúde da família [...]." (Gestor, AB, Município A)

"Eu acho que são os dois na verdade (os médicos da $A B$ e da $A E$ ). Não tem como responsabilizar uma pessoa só..." (Médica, AB, Município A)

No município $B$, verificou-se que os profissionais da Atenção Básica (AB) são vistos como responsáveis pelo seguimento do paciente entre os níveis de atenção. Essa atribuição recai, segundo alguns entrevistados, sobre a equipe, de modo indistinto.

"Eu acho que se ele tem uma atenção básica, tem a equipe de PACS/PSF. Eu acho que toda equipe é multiprofissional, todos eles são responsáveis pela paciente." (Enfermeira, $A E$, Município B)

Para os gerentes e médicos da Atenção Especializada (AE), essa responsabilidade caberia a determinadas categorias profissionais da AB.

“Eu acho que o responsável mesmo seria o médico da atenção básica, porque ele vai ver o paciente como um todo. Ele vai receber as informações das outras especialidades que forem necessárias. É o que estaria, assim, mais capacitado para ver o paciente como um todo." (Médica, AE, Município B)

“É o enfermeiro do PSF e do PACs. [...] existe o médico, mas o PACs não tem o médico. Então a autonomia na equipe é o enfermeiro, [...] mesmo sendo PSF, dificilmente é o profissional médico; quem faz a ponte toda de ação é o enfermeiro." (Gerente, AE, Município B)

A exemplo do município $A$, os relatos manifestam mais a visão dos entrevistados acerca de quem deveria ter a responsabilidade clínica, do que a identificação da presença efetiva de um profissional que mantenha o acompanhamento do paciente enquanto este transita entre os níveis.

Para Starfield ${ }^{7}$, os profissionais da $A B$ seriam os mais indicados para se responsabilizarem pelo percurso terapêutico do usuário na maior parte dos episódios de adoecimento. Outros autores mostram-se reticentes a esse respeito, duvidando que o modelo de APS vigente possa dar conta de funções como esta e outras relacionadas à ordenação de fluxos na rede ${ }^{29,30}$. Defendem a ampliação da capacidade resolutiva como forma de abranger o cuidado comunitário e certos procedimentos, antes exclusivos da atenção de segundo e terceiro nível, como: pequenas cirurgias, diagnose, urgências, dentre outros. Além disso, seriam necessários mais recursos e programas regulares de educação continuada para lhes dar maior prestígio e reconhecimento.

$A$ insuficiência de comunicação entre $A B$ e $A E$ como parte da dinâmica de seguimento do paciente é justificada de diferentes formas pelos entrevistados, sendo apresentada como causa e consequência de problemas que dificultam a coordenação da atenção entre níveis.

No município $B$, observou-se a percepção de profissional da $A E$ que, uma vez recebido um encaminhamento, assume a condução do caso. Entende que, a partir de então, o contato com a $A B$ ficaria destituído de qualquer utilidade prática, assim como o retorno do paciente para seguimento na unidade básica (UBS).

“[...] o paciente acaba ficando com a gente, entende? [...] eu não vou devolver o doente. Do ponto de vista do diabetes ele é meu agora. Não foi encaminhado? É como se, na minha 
opinião assim, lá não estivessem conseguindo compensar o doente e mandaram para cá, então eu vou ficar acompanhando." (Médica, AE, Município B)

Profissionais que atuam em postos de gestão e gerenciamento nessa rede apresentam, como causa dessa dificuldade, a falta de confiança dos especialistas nos colegas da $A B$, os quais, dentre outras coisas, manteriam as prescrições do especialista, atualizando tão somente as datas das receitas.

"E a gente identifica que a referência e a contrarreferência não acontecem a contento porque muitos médicos da média complexidade não confiam na atenção básica. Aí o paciente chega aqui, numa unidade de média complexidade, e se vincula a ela e não sai mais. [...] ou ele acha muito cômodo ficar atualizando receita, enfim, fazendo um trabalho meramente burocrático, entendeu?!" (Gerente, AE, Município B)

É possível verificar a relação entre as dimensões 'seguimento' e 'coerência', esta última correspondendo à existência de aproximações e objetivos comuns de tratamento por parte dos profissionais dos diferentes níveis de atenção ${ }^{20,28}$. Isso visa a assegurar a pertinência da transferência do paciente de um nível a outro, bem como a inexistência de duplicação de exames, de consultas e de contraindicação de medicamentos.

Os médicos especialistas relataram encaminhamentos desnecessários feitos pelos médicos da $A B$, gerando deslocamentos inúteis dos pacientes e acompanhantes, e subtração de vagas da $A E$ que poderiam ser disponibilizadas a pacientes com indicação precisa. Também citam encaminhamentos feitos pela $A B$ para unidades especializadas que não têm perfil assistencial para a resolução do caso.

“Eu vou mandar para lá, pelo menos ele é visto pelo endócrino; não vai resolver, mas vai ser visto pelo endócrino (pensamento atribuído ao médico da $A B$ )..., ou seja, aquele paciente que eu fiquei avaliando para dizer que não era daqui, deixei de atender um diabético que precisa ir para insulinoterapia, porque não conseguiu vaga." (Médica, $A E$, Município B)

Tal questão também emergiu das falas dos entrevistados do município $A$, onde um médico especialista contradiz a opinião de um colega da $A B$ que entende existir coerência da atenção entre níveis. $O$ especialista informa que essa aparente concordância ocorre porque o médico da $A B$ não assume outra conduta a não ser a de encaminhar para a $A E$.

“[...] Geralmente, o médico da atenção básica ele se exime do problema, [...] para não se responsabilizar, muitas vezes, ele fica mantendo só o que o especialista disse que é, como se ele tivesse a chancela do especialista para lhe dar mais garantia. Então, dificilmente tem contradição." (Médico, AE, Município A)

Referências às tensões e estranhamentos entre generalistas e especialistas, citados como o que dificultam a coordenação, aparecem tanto nos relatos sobre sistemas de atenção à saúde em outros países como nos textos que abordam o caso brasileiro, embora não seja um tema fartamente estudado com a profundidade merecida ${ }^{27,18}$. Os autores levantam explicações para o fato, e uma delas diz respeito à cultura estabelecida no meio médico segundo a qual o conhecimento do generalista estaria sempre aquém do especialista, concepção amplamente refutada por McWhinney e Freeman ${ }^{31}$ como sendo um equívoco epistemológico.

Apoiadores institucionais que atuam no município A identificam duplicações de procedimentos de natureza diagnóstica e terapêutica, problema citado como bastante recorrente no que diz respeito à prescrição medicamentosa:

"[...] eu acho que o paciente acaba sendo mal conduzido, porque cada um vê o paciente de uma forma e, muitas vezes, pode até ser que repita o que o paciente já esteja fazendo em um serviço e o outro acaba prescrevendo a mesma coisa, muitas vezes, sem ter esse conhecimento 
mais aprofundado, sem ter nada por escrito, nenhum papelzinho que realmente prove que $\mathrm{o}$ paciente está fazendo uso." (Apoio institucional, AE, Município A)

No município $B$, houve relatos que demonstram a falta de reconciliação de tratamentos, outro atributo da coerência entre os níveis de atenção ${ }^{20,28}$. Profissionais da $A B$ revelaram mudanças feitas em suas prescrições pelos médicos da $A E$, substituindo nomes comerciais dos medicamentos, ainda que mantendo os mesmos fármacos. Se o procedimento não for devidamente explicado ao paciente, este ficará com a impressão de que a conduta do médico da $A B$ está sendo modificada, o que pode confundi-lo e deixá-lo inseguro em relação ao acompanhamento do generalista.

Por outro lado, a reconciliação de tratamentos também não é cogitada pelos especialistas, que, ao observarem divergências entre condutas, insistem que o paciente deve, simplesmente, desconsiderar a prescrição feita pelo médico da $A B$.

"Então eu faço uma receita, o médico da atenção básica modifica e enfim, não há uma comunicação, não há uma ajuda. Então, o próprio paciente fica meio perdido, porque não sabe quem seguir. Então, eu acho que não há atividades complementares; ficam os dois disputando por uma conduta, uma prescrição e, inclusive, eu sou bem enfática: "olhe em relação ao diabetes, siga a minha orientação! Né! Eu sou especialista!" (Médica, AE, Município B)

Os erros no manejo dos casos e na referência para a $A E$ levantados na pesquisa pelos especialistas poderiam ser debitados na conta do modelo de formação dos médicos no Brasil, assunto abordado por Mendes $^{32}$ e McWhinney e Freeman ${ }^{31}$, que destacam a necessária mudança curricular e metodológica, propiciando, desde a graduação, vivências para uma formação profissional generalista com uma visão ampliada da clínica e senso de responsabilidade social.

Martinez et al. ${ }^{26}$ abordam a questão a partir da separação histórica entre as diferentes práticas profissionais e da forma fragmentada como até hoje são formados os trabalhadores da saúde, o que contribuiria para que certas categorias se sentissem senhoras de todo o processo assistencial, com resistência a rever seus valores.

Quanto à 'acessibilidade', dimensão que se refere à garantia de atenção sem interrupções entre níveis assistenciais de acordo com a necessidade do paciente ${ }^{20,28}$, emergiu, em ambos os municípios, como sujeita a sérias dificuldades.

O problema, no entanto, não foi referido para todas as especialidades com a mesma intensidade, respondendo indistintamente à disponibilidade de especialistas atuando no território das redes municipais de saúde.

No município A, muitos pacientes não estariam conseguindo atendimento em tempo oportuno, permanecendo em filas de espera no sistema de regulação, o que interrompe sua trajetória assistencial.

“[...] tem gente que chegou a dizer que já esperou quase três anos para ter uma consulta de 15 minutos comigo aqui!" (Médico, AE, Município A)

Essa dificuldade de acessar a AE é vista como associada à desproporção entre oferta e demanda para determinadas especialidades.

“[...] a referência para o nível secundário, ela em alguns casos... é, vamos dizer assim, não tão difícil. Como por exemplo, encaminhamento para endocrinologista não é tão difícil, porque a quantidade que é encaminhada é pequena. Pneumologista também não é tão difícil. Eu acredito que, em torno de um mês, se consigam as consultas com esses especialistas. Mas, já outros tipos de referência, como, por exemplo, oftalmologista, psiquiatra... aí complica mais. Às vezes, não se consegue mesmo." (Médico, AB, Município A)

Além da necessidade de ampliação da oferta de procedimentos especializados, outra causa já citada pelos especialistas seria o encaminhamento desnecessário para consultas e exames no segundo 
nível da atenção, gerando sobrecarga de demanda. No entanto, para os médicos da $A B$, uma maior autonomia dos médicos generalistas para o manejo de certos casos seria uma condição redutora do número de encaminhamentos para $\mathrm{AE}$.

A restrição imposta aos médicos da $A B$ para solicitação de determinados exames também é apresentada como uma das causas. A insuficiência do número de vagas para consultas especializadas é atribuída, ainda, ao descumprimento dos horários de trabalho por parte dos profissionais.

Ainda sobre a relação desfavorável entre oferta e demanda por consulta especializada, a pesquisa registra uma única opinião a respeito do descumprimento de horários por parte de médico especialista, condição que não pode ser desprezada, uma vez que se trata de problema tão conhecido quanto silenciado pelas equipes de saúde e gerentes, ao qual a literatura disponível tem dedicado pouco espaço, limitando-se à apresentação e discussão dos aspectos normativos.

Estudos realizados em município do nordeste brasileiro também identificaram problemas na continuidade da atenção, como: a ausência de acolhimento para organizar a demanda segundo critérios de necessidades clínicas; falta de médicos nas USF; agendamento de consultas segundo distribuição de senhas; ausência de estratégias e baixa resolubilidade ${ }^{33}$. Tais aspectos contribuem para dificultar o acesso da população que procura os serviços e o atendimento oportuno para os problemas de saúde ${ }^{34}$.

Embora o aumento da capacidade instalada seja visto, pelos que ocupam cargos de gestão e gerenciamento, como resposta aos problemas de coordenação, tal solução é aceita apenas em parte por Solla e Paim ${ }^{35}$. Para esses autores, a expansão desses números tem como motivação as necessidades de procedimentos especializados decorrentes do aumento de cobertura da $A B$, onde as solicitações nem sempre obedecem a processos e instrumentos regulatórios efetivos, a exemplo da utilização de protocolos assistenciais.

Problemas de acessibilidade também foram citados como causas de complicações do estado de saúde do paciente.

"O paciente fica perdido nesse caminho, ele vai ficar indo e voltando e, às vezes, pode culminar com um agravamento da doença. Se for uma doença crônica, ele pode vir a óbito, e não é por falta de assistência. [...]" (Apoio institucional, AB, Município B)

Tais complicações são reportadas dessa forma por profissionais de saúde que atuam no município A:

“[...] a gente tem muitos diabéticos, muitos deles já com vasculopatia ou neuropatia diabética; a gente tenta encaminhar, mas não consegue. Então a gente termina ficando com esse paciente, esperando uma complicação, porque a vasculopatia é uma complicação. Então, a gente espera a complicação maior, uma úlcera, uma isquemia para conseguir essa vaga." (Médico, AB, Município A)

Os entrevistados que atuam no Município B informam que os problemas de acessibilidade não se restringem às consultas e exames, mas, também, à dispensação dos medicamentos.

“[...] então, muitas vezes, ele precisa de um encaminhamento para um tratamento, mas não consegue, a medicação não está disponível, estamos com falta de alguma medicação no sistema central; então, tudo isso interfere para a qualidade da assistência." (Apoio institucional, $A B$, Município B)

É inegável, portanto, a frequência e recorrência com que a acessibilidade é citada pelos entrevistados, assumindo o status de principal elemento a dificultar uma efetiva coordenação da atenção entre níveis. Almeida et al. ${ }^{8}$ destacam, em seu artigo sobre a coordenação do cuidado em quatro capitais brasileiras, que a descentralização da atenção secundária com organização de fluxos assistenciais é uma das estratégias para ampliar o acesso e a integração da rede, facilitando o acesso em tempo oportuno e a diminuição de barreiras financeiras para os usuários. 
A percepção dos entrevistados revela tanto a existência de problemas na coordenação da atenção como o esforço no sentido de conter a fragmentação da rede. Com diferentes níveis de evolução, tal fato tem sido observado no Brasil e em outros países do mundo, não se constituindo em problema novo. Silva ${ }^{36}$, citando um documento da Organização Panamericana de Saúde sobre redes integradas de serviços, reforça que o problema já se constituía em preocupação para os países desenvolvidos desde as primeiras décadas do século passado, conforme demonstra o Relatório Dawson, de 1920.

A centralidade que o tema vem ocupando nas últimas décadas em todo o mundo deve-se à elevação da demanda de doentes crônicos e à necessidade de otimização entre custo e eficácia na prestação da atenção. Na Europa, as reformas pró-coordenação vêm ocorrendo desde a década de 1990, sendo estas um dos motivos pelos quais a produção científica a respeito é bem mais alentada do que na América Latina, onde as reformas a esse respeito são ainda incipientes ${ }^{8}$.

No caso do Brasil, Silva ${ }^{36}$ comenta que a integralidade, finalidade última da coordenação da atenção, é um elemento fundamental da construção do SUS, mas talvez seja um dos seus pilares mais frágeis. Mostra-se onipresente em suas bases legais, embora sua efetivação enfrente sérias dificuldades de natureza política, administrativa, financeira e até cultural.

Em que pese o porte dos municípios estudados e das redes de saúde que eles coordenam e operam, não foi identificada, pelos entrevistados, a prática de iniciativas visando à aproximação dos profissionais da $A B$ e da $A E$ com o objetivo de: promover o conhecimento mútuo, o intercâmbio para fortalecer a qualificação profissional, a troca de informações sobre o paciente e o manejo conjunto de casos. Há, portanto, uma clara necessidade de implantar ou melhorar os mecanismos de coordenação da rede com base em comunicação direta entre os profissionais, tais como: sistema expert, grupos de trabalho multidisciplinares, instrumentos para comunicação informal. Tais mecanismos, além de melhorarem o diagnóstico e monitoramento da capacidade da $A B$, servem para criar espaços para estreitar as relações diretas entre os profissionais ${ }^{27,37}$.

\section{Conclusões}

Os achados da pesquisa aqui relatada vêm contribuir para a produção de conhecimento sobre a coordenação entre níveis de atenção à saúde a partir das opiniões dos profissionais que atuam em dois municípios de grande porte da Região Nordeste do Brasil.

É importante destacar que o incipiente quantitativo de estudos no Brasil com foco nesse objeto e sobre os fatores que o influenciam dificulta a comparação dos achados. Acrescente-se o fato de que as metodologias dessas investigações diferem entre si, seguindo, com frequência, a linha dos estudos quantitativos. Essas limitações devem ser tomadas em conta na interpretação dos resultados e na sua transferência para outros contextos.

Ainda assim, é possível verificar que algumas dificuldades são comuns, como: a de se obter um efetivo exercício da referência e contrarreferência e do acesso aos exames, sobretudo os de imagem, bem como de consultas em determinadas especialidades.

A exemplo de outras pesquisas, é patente o reconhecimento da importância da coordenação pelos informantes e o esforço que alegam investir nesse processo. Ao mesmo tempo, fica demonstrado que os profissionais envolvidos enxergam a coordenação como algo ainda distante do cotidiano das redes.

Também ficou evidenciada a tensão verificada entre médicos da $A B$ e da $A E$ pelas críticas de lado a lado. O desenvolvimento de mecanismos de aproximação entre essas categorias, visando a um trabalho cooperativo, não foi registrado pelos entrevistados.

Um achado que não é comum nas pesquisas nacionais diz respeito ao aprofundamento das causas dessa difícil convivência profissional, gerando razões que indicam a necessidade de estudos dirigidos para identificar a possível interferência, nessa realidade, de fatores ligados: ao mercado de trabalho, à formação acadêmica e aos valores que definem a representação social acerca do generalista e do especialista. São fatores que desafiam a eficácia de medidas mais imediatas, como o uso de tecnologias facilitadoras da comunicação de informação sobre os pacientes. 


\section{Colaboradores}

Renata Patrícia Freitas Soares de Jesus: coordenou e participou da coleta dos dados, da análise dos dados e da redação do artigo; participou ativamente da discussão dos resultados; participou da revisão e aprovação da versão final do trabalho. Antônio Carlos Gomes do Espírito Santo: participou da análise dos dados e da redação do artigo; participou ativamente da discussão dos resultados; participou da revisão e aprovação da versão final do trabalho. Marina Ferreira de Medeiros Mendes: participou da coleta dos dados, da análise dos dados e da redação do artigo; participou ativamente da discussão dos resultados; participou da revisão e aprovação da versão final do trabalho. Isabella Chagas Samico: participou da elaboração do projeto, da coleta dos dados, da análise dos dados e da redação do artigo; participou ativamente da discussão dos resultados; participou da revisão e aprovação da versão final do trabalho.

\section{Referências}

1. Organização Panamericana de Saúde. Renovação da Atenção Primária em Saúde nas Américas. Washington, DC: WHO; 2005.

2. World Health Organization. Primary Health Care. Now more than ever. Geneva: WHO; 2008. The World Health Report 2008.

3. Haggerty JL, Yavich N, Báscolo EP. Grupo de consenso sobre un marco de evaluación de la atención primaria en américa latina. Un marco de evaluación de la atención primaria de salud en América Latina. Rev Panam Salud Publica. 2009; 26(5):377-84.

4. Haggerty JL, Reid RJ, Freeman GK, Starfield BH, Adair CE, McKendry R. Continuity of care: a multidisciplinaryreview. BMJ. 2003; 327:1219-21.

5. Núñez RT, Lorenzo IV, Naverrete ML. La coordinación entre niveles asistenciales: una sistematización de sus instrumentos y medidas. Gac Sanit. 2006; 20(6):485-95.

6. Almeida PF, Giovanella L, Nunan BA. Coordenação dos cuidados em saúde pela atenção primária à saúde e suas implicações para a satisfação dos usuários. Saude Debate. 2012; 36(94):375-91.

7. Starfield B. Atenção primária - equilíbrio entre necessidades de saúde, serviços e tecnologia. Brasília: UNESCO, Ministério da Saúde; 2002.

8. Almeida PF, Giovanella L, Mendonça MHM, Escorel S. Desafio à coordenação dos cuidados em saúde: estratégias de integração entre níveis assistenciais em grandes centros urbanos. Cad Saude Publica. 2010; 26(2):286-98.

9. Malta DC, Merhy EE. O percurso da linha do cuidado sob a perspectiva das doenças crônicas não transmissíveis. Interface (Botucatu). 2010; 14(34):593-605.

10. Mendes EV. As redes de atenção à saúde. Brasília, DF: OPAS; 2011.

11. Mendes EV. As redes de atenção à saúde. Cienc Saude Colet. 2010;15(5):2297-305.

12. Terraza-Núñez R, Vargas I, Vázquez ML. La coordinación entre niveles asistenciales: una sistematización de sus instrumentos y medidas. Gac Sanit. 2006; 20(6):485-95.

13. Vázquez ML, Vargas I, Unger JP, Mogollón AS, Silva MR, Paepe P. Integrated health care networks in Latin America: toward a conceptual framework for analysis. Rev Panam Salud Publica. 2009; 26(4):360-7.

14. Vargas I, Vázquez ML, Henao D, De Campos C. Implantación de mecanismos de coordinación asistencial en Redes Integradas de Servicios de Salud. Barcelona: Consorci de Salut i Social de Catalunya (CSC), OPS; 2011.

15. World Health Organization. Global status reporton non-communicable diseases 2014. Geneva: WHO; 2014. 
16. Conill EM, Fausto MCR, Giovanella L. Contribuições da análise comparada para um marco abrangente na avaliação de sistemas orientados pela atenção primária na América Latina. Rev Bras Saude Mater Infant. 2010; 10(1):14-27.

17. Ministério da Saúde (BR). Secretaria Executiva. Departamento de Apoio à Descentralização. Coordenação-Geral de Apoio à Gestão Descentralizada. Diretrizes operacionais dos pactos pela vida, em defesa do SUS e de gestão. Brasília: Ministério da Saúde; 2006.

18. Giovanella L. Redes integradas, programas de gestão clínica e generalista coordenador: análise das reformas recentes do setor ambulatorial na Alemanha. Cienc Saude Colet. 2011;16(1):1081-96.

19. Escorel S, Giovanella L, Mendonça MHM, Senna MCM. O Programa de saúde da família e a construção de um novo modelo para a atenção básica no Brasil. Rev Panam Salud Publica. 2007; 21:164-76.

20. Vázquez ML, Vargas I, Unger JP, De Paepe P, Mogollón-Pérez AS, Samico I, et al. Evaluating the effectiveness of care integration strategies in diferent healthcare systems in Latin America: the EQUITY-LA II quasi-experimental study protocol. BMJ Open. 2015; 1-5.

21. Instituto Brasileiro de Geografia e Estatística - IBGE. Indicadores sociais municipais. Rio de Janeiro: IBGE; 2011.

22. Gilson L, editor. Health policy and systems research: a methodology reader. Geneva: WHO, Alliance for Health Policy and System Research; 2012. p. 19-39.

23. Saltmann RB, Rico A, Boerma W, editors. Primary care in the driver's seat? Organizational reform in European primary care. European observatory on health systems and policies series. Berkshire: Open University Press; 2006.

24. Mendes EV. Os grandes dilemas do SUS. Salvador: Casa da Qualidade; 2001.

25. Ministério da Saúde (BR). Portaria n².488, de 21 de outubro de 2011. Aprova a Política Nacional de Atenção Básica, estabelecendo a revisão de diretrizes e normas para a organização da Atenção Básica, para a Estratégia Saúde da Família (ESF) e o Programa de Agentes Comunitários de Saúde (PACS). Diário Oficial da União. 21 Out 2011.

26. Martinez DH, Navarrete MLV, Lorenzo IV. Factores que influyen en la coordinación entre niveles asistenciales según la opinión de directivos y profesionales sanitarios. Gac Sanit. 2009; 23(4):280-6.

27. Vargas I, Mogollón-Pérez AS, De Paepe P, Ferreira da Silva MR, Unger JP, Vázquez $M L$. Barriers to healthcare coordination in market-based and decentralized public health systems: a qualitative study in healthcare networks of Colombia and Brazil. Health Policy Plan. 2016; 31(6):736-48.

28. Aller MB, Vargas I, Codersh J, Calero S, Cots F, Abizanda $M$, et al. Development and testing of indicators to measure coordination of clinical information and management across levels of care. BMC Health Serv Res. 2015; 15:323.

29. Almeida PF, Fausto MCR, Giovanella L. Fortalecimento da atenção primária à saúde: estratégia para potencializar a coordenação dos cuidados. Rev Panam Salud Publica. 2011; 9(2):84-95.

30. Watson DE, Broemeling AM, Reid RJ, Black CA. Results-based logic model for primary health care: laying an evidence-based foundation to guide performance measurement, monitoring and evaluation. Vancouver: Centre for Health Services and Policy Research, University of British Columbia; 2004.

31. McWhinney I, Freeman T. Manual de medicina da família e comunidade. 3a ed. Porto Alegre: Artmed; 2010. 
32. Mendes EV. O cuidado das condições crônicas na atenção primária à saúde: o imperativo da consolidação da estratégia da saúde da família. Brasília, DF: OPAS; 2012.

33. Sousa FOS, Medeiros KR, Gurgel Júnior GD, Albuquerque PC. Do normativo à realidade do Sistema Único de Saúde: revelando barreiras de acesso na rede de cuidados assistenciais. Cienc Saude Colet. 2014; 19(4):1283-93.

34. Travassos C, Castro MSM. Determinantes e desigualdades sociais no acesso e na utilização de serviços de saúde. In: Giovanella L, Escorel S, Lobato LVC, Noronha JC, Carvalho Al, organizadores. Políticas e sistemas de saúde no Brasil. Rio de Janeiro: Fiocruz, Cebes; 2008. p. 215-45.

35. Solla JJSP, Paim JS. Relação entre a atenção básica, de média e alta complexidade: desafios para organização do cuidado no Sistema Único de Saúde. In: Paim JS, AlmeidaFilho N, organizadores. Saúde Coletiva: teoria e prática. Rio de Janeiro: MedBook; 2014. p. 343-52.

36. Silva SF. Organização das redes regionalizadas e integradas de atenção à saúde: desafios do Sistema Único de Saúde (Brasil). Cienc Saude Colet. 2011; 16(6):2753-62.

37. Carneiro MSM, Melo DMS, Gomes JM, Pinto FJM, Silva MGC. Avaliação do atributo coordenação da Atenção Primária à Saúde: aplicação do PCATool a profissionais e usuários. Saude Debate. 2014; 38:279-95.

Jesus RPFS, Espírito Santo ACG, Mendes MFM, Samico IC. Percepción de los profesionales sobre la coordinación entre niveles de atención a la salud en dos municipios de gran porte del Estado de Pernambuco, Brasil. Interface (Botucatu). 2018; 22(65):423-34.

El objetivo fue conocer la percepción de profesionales de salud sobre la coordinación de la atención entre niveles asistenciales en dos municipios del Estado de Pernambuco, Brasil. Se realizó encuesta cualitativa, realizada por medio de entrevistas en profundidad, con 51 profesionales de atención básica $(A B)$ y especializada $(A E)$ en relación a la coordinación de la gestión clínica y factores que influyen sobre ella. Los resultados mostraron que, a pesar de observarse una comprensión insuficiente del término Coordinación de la atención entre niveles, hay un reconocimiento de la importancia y del esfuerzo de la inversión en ese proceso. La insuficiencia de la comunicación entre $A B$ y AE es vista como causa y consecuencia de problemas que dificultan la coordinación de la gestión clínica en las dimensiones del seguimiento, capacidad de acceso y coherencia de la atención. No se observaron iniciativas de aproximación entre profesionales de la $A B$ y de la $A E$ en el sentido de promover el conocimiento mutuo, fortalecer la calificación profesional y el manejo conjunto de los casos.

Palabras clave: Atención a la salud. Niveles de atención a la salud. Gestión clínica. Encuesta cualitativa. 